\title{
Cellular Antenna Location Optimization in Distributed MIMO Systems
}

\author{
Man Sun ${ }^{1, a}$, Yueheng $\mathrm{Li}^{2, \mathrm{~b}}$, Tuanjie Xue, Ping Huang, Feng Wang \\ ${ }^{1}$ College of Computer and Information, Hohai University, Nanjing, Jiangsu, 211100, China \\ ${ }^{2}$ College of Computer and Information, Hohai University, Nanjing, Jiangsu, 211100, China \\ aemail:1762637201@qq.com, bemail:yueheng_li@hhu.edu.cn
}

Keywords: Distributed MIMO, antenna location optimization, ergodic capacity, PSO algorithm

\begin{abstract}
In this paper, the optimization of antenna location of a distributed MIMO (DMIMO) systems in a circular cell based on advanced particle swarm optimization(PSO) algorithm is analyzed.Firstly, a composite fading channel including small scale fading,path loss and shadow fading is established;then, a closed form expression of downlink average ergodic capacity under highersignal-to-noise (SNR) condition is deduced;next,the optimal position of antenna ports in the circular cell is quickly searched by PSO through dividing the cell into several predefined areas. Simulation results show that the searched position of antenna ports in the cell is very close to the optimaltheoretical location.
\end{abstract}

\section{Introduction}

Due to the rapid development of radio communications, the research of distributed MIMO (DMIMO) system has become a hot issue because of its wide coverage, spectrum efficiency, and etc. Compared with traditional centralized MIMO (CMIMO) systems, antenna ports of DMIMO systems can be distributed in different geographical position of the cell to reduce the average access distance between user terminal and antenna ports[1]. For DMIMO systems, the antenna location in a cell will have an important effect on the whole system's performance[2].

Current researches on the optimal position distribution of multiple antennas in DMIMO systems have achieved preliminary results. The optimal antenna position in a linear cell was analyzed by using average bit error rate as an optimization object[3]. When both transmitter and receiver sides are equipped with multiple antennas, maximum output SNR was obtained by using maximal ratio combining (MRC) technique[4]. By signal transmission adopting selective transmission scheme (ST), [5] used K-means algorithm to optimize the antenna position in a single and in double cells respectively. Because the number of antennas is limited to just one for base station and user mobile, and the intelligent genetic algorithm used in [6] has a lower convergence rate, it is impossible to search the antenna port position quickly in cells of future complex DMIMO systems.

This paper focuses on the cellular system of mobile communication which sets a simplified circular cell model that adopts ST at distributed antenna port and MRC at receiver side. Downlink average ergodic capacity is deduced and regarded as the system optimization target.

\section{System Model}

As shown in Fig.1, we consider a circular cell of DMIMO systems with radius $R$, which consists of $N$ distributed antenna ports (DAU); one of them in the center of the cell is fixed and regarded as central processing unit (CPU) or central antenna port (DAU1), and the other DAUs and mobile station (MS) which is equipped with $M$ antennas are distributed anywhere. All DAUs are equipped with $L$ antennas; $(\rho, \theta)$ and $\left(r_{i}, \beta_{i}\right)(i=1,2, \ldots, N)$ represent MS and DAUi's polar coordinate respectively. The circular cell of DMIMO systems is denoted as $(M, N, L)$-DMIMO. 


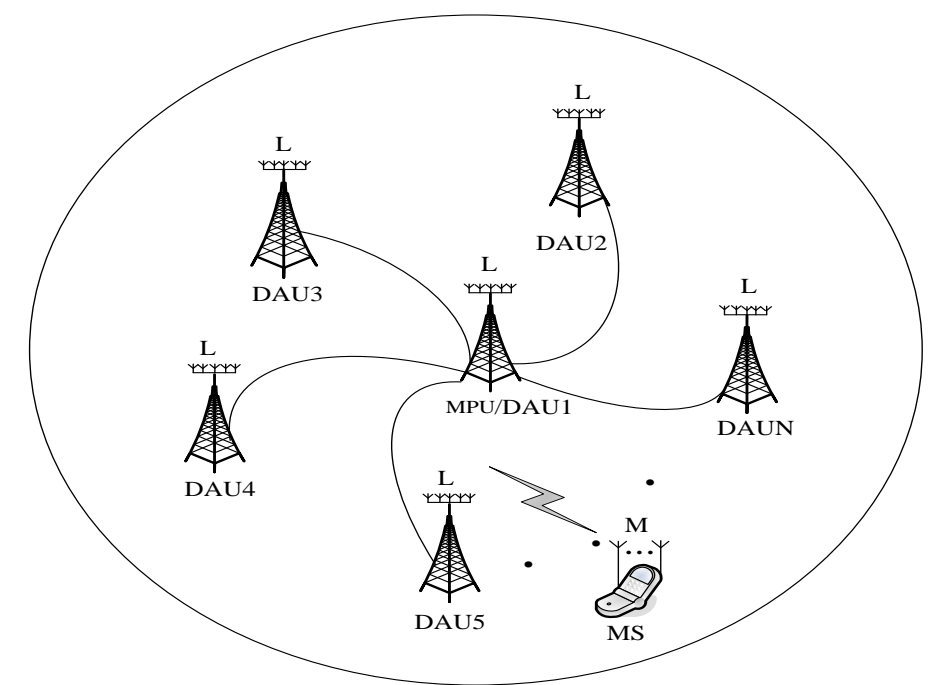

Fig.1. Illustration of a circular DMIMO system

For simplicity, a flat Rayleigh fading is assumed to be the small-scale fading in the composite channel model. In downlink, the transmitter adopts ST scheme that selects a DAU with the best channel condition. Therefore, the received signal can be expressed as

$$
\mathbf{y}=\sqrt{P_{t}} \mathbf{H}_{i} \mathbf{w} x+\mathbf{n},
$$

where $\mathbf{y}=\left[y_{1}, \mathrm{~L}, y_{M}\right]^{T}$ is received signal vector; $x$ is transmitted symbol and $E\left[|x|^{2}\right]=1 ; \mathbf{H}_{i}$ is an $M \times L$ channel matrix of $\mathrm{DAU}_{\mathrm{i}}$. Considering a small and large scale fading, the composite channel model between MS and DAUi can be established as

$$
\mathbf{H}_{i}=\sqrt{\frac{c s_{i}}{d_{i}^{\alpha}}} \mathbf{g}_{i}, \quad 1 \leq i \leq N(2)
$$

In above formula, from [7], $\mathrm{c}=\gamma R^{\alpha} / P_{t}$, where $\gamma$ represents transmit SNR; $\mathbf{g}_{i}$ represents small scale fading matrix with parameter $m_{i} ; s_{i}$ represents shadow fading with zero mean and variance $\sigma_{s h}^{2}, 10 \log _{10} s_{i} \sim\left(0, \sigma_{s h}{ }^{2}\right) ; \alpha$ is path loss exponent; $d_{i}$ is the distance between MS and DAUi and the expression of $d_{i}$ can be

$$
d_{i}=\sqrt{\rho^{2}+r_{i}^{2}-2 \rho r_{i} \cos \left(\theta-\beta_{i}\right)}(3)
$$

Output signal when the receiver adopt MRC can be denoted as:

$$
\mathbf{z}=\left(\mathbf{H}_{i} \mathbf{w}\right)^{H} \mathbf{y}=\sqrt{\mathrm{P}_{\mathrm{t}}}\left(\mathbf{H}_{i} \mathbf{w}\right)^{H}\left(\mathbf{H}_{i} \mathbf{w}\right) x+\left(\mathbf{H}_{i} \mathbf{w}\right)^{H} \mathbf{n}(4)
$$

Then, the output SNR has the following expression:

$$
S N R_{i}=P_{t}\left(\mathbf{H}_{i} \mathbf{w}\right)^{H}\left(\mathbf{H}_{i} \mathbf{w}\right)=P_{t}\left(\sum_{p=1}^{L} \sum_{q=1}^{L} w_{p} w_{q}^{*} \sum_{k=1}^{M} h_{k p} h_{k q}^{*}\right)(5)
$$

From [8], when $\left(w_{p} w_{q}^{*}\right)^{*}=\sum_{k=1}^{M} h_{k p} h_{k q}^{*} /\left|\sum_{k=1}^{M} h_{k p} h_{k q}^{*}\right|$, output SNR is the largest, and then the maximum output SNR is:

$$
S N R_{i}=P_{t}\left(\sum_{p=1}^{L} \sum_{q=1}^{L}\left|\sum_{k=1}^{M} h_{k p} h_{k q}^{*}\right|\right)
$$

\section{Analysis of Cellular Capacity}

When transmitter adopts ST, the antenna port with the largest output SNR is as the final output port. Therefore, downlink point-to-point ergodic capacity expression is 


$$
C(\rho, \theta)=\mathrm{E}_{\mathbf{H}}\left\{\log _{2} \operatorname{det}\left(\mathbf{I}_{K}+\frac{P_{\mathrm{t}}}{L} \mathbf{H}_{m}{ }^{H} \mathbf{H}_{m}\right)\right\}(7)
$$

where, $\mathbf{I}_{K}$ represents Kth order identity matrix, $K=\min (L, M)$; $\operatorname{det}(g)$ represents determinant. According to different number of transmitter and receiver antennas, the point to point downlink ergodic capacity when $M>L$ and $M \leq L$ respectively under high SNR is analyzed as below[9] .

(1) $M>L$

$$
C(\rho, \theta)=\mathrm{E}_{\mathbf{H}}\left\{\log _{2} \operatorname{det}\left(\mathbf{I}_{L}+\frac{\mathrm{P}_{\mathrm{t}}}{L} \mathbf{H}_{m}{ }^{H} \mathbf{H}_{m}\right)\right\}=\mathrm{E}_{s, g}\left\{\log _{2} \operatorname{det}\left(\mathbf{I}_{L}+\frac{\gamma}{L} \Omega_{\mathbf{g}_{m}}{ }^{H} \mathbf{g}_{m}\right)\right\}
$$

Where $\Omega=\left(R / d_{m}\right)^{\alpha} s_{m}, \quad \gamma=\mathrm{cP}_{\mathrm{t}} / R^{\alpha}$. From $\operatorname{det}(\mathbf{I}+\mathbf{A B})=\operatorname{det}(\mathbf{I}+\mathbf{B A})$, the above equation (8) can be rewritten as

$$
C(\rho, \theta)=\mathrm{E}_{s, g}\left\{\log _{2} \operatorname{det}\left(\mathbf{I}_{L}+\frac{\gamma}{L} \Omega \mathbf{g}_{m} \mathbf{g}_{m}{ }^{H}\right)\right\}
$$

The above formula can ignore the effect of identity matrix $\mathbf{I}$, and is approximate to be

$$
\begin{aligned}
C(\rho, \theta) & \cong \mathrm{E}_{s, g}\left\{\log _{2} \operatorname{det}\left(\frac{\gamma}{L} \Omega \mathbf{g}_{m} \mathbf{g}_{m}{ }^{H}\right)\right\} \\
& =L \log _{2}\left(\frac{\gamma}{L}\right)+\mathrm{E}_{s}\left\{\log _{2} \operatorname{det}(\Omega)\right\}+\mathrm{E}_{g}\left\{\log _{2} \operatorname{det}\left(\mathbf{g}_{m} \mathbf{g}_{m}{ }^{H}\right)\right\}
\end{aligned}
$$

Due to $\operatorname{det}(\Omega)=\left[\left(\frac{R}{d_{m}}\right)^{\alpha} s_{m}\right]^{L}$ and $\mathrm{E}\left(\ln s_{m}\right)=0, \quad$ besides $\left(\mathbf{g}_{m} \mathbf{g}_{m}{ }^{H}\right)$ undergoes Wishart distribution according to theorem 2.11 in [10], where $\psi(\cdot)$ is digamma function, (10) is deduced

$$
C(\rho, \theta) \cong L \log _{2}\left(\frac{\gamma}{L}\right)+L \log _{2}\left(\frac{R}{d_{m}}\right)^{\alpha}+\frac{1}{\ln 2} \sum_{i=0}^{L-1} \psi(M-i)
$$

(2) $M \leq L$

$$
\begin{aligned}
C(\rho, \theta) & =\mathrm{E}_{\mathbf{H}}\left\{\log _{2} \operatorname{det}\left(\mathbf{I}_{M}+\frac{\mathrm{P}_{\mathrm{t}}}{L} \mathbf{H}_{m} \mathbf{H}_{m}{ }^{H}\right)\right\} \\
& =\mathrm{E}_{s, g}\left\{\log _{2} \operatorname{det}\left(\mathbf{I}_{M}+\frac{\gamma}{L}\left(\frac{R}{d_{m}}\right)^{\alpha} s_{m} \mathbf{g}_{m} \mathbf{g}_{m}{ }^{H}\right)\right\}
\end{aligned}
$$

Sme to $M>L$ deduction process, equation (12) is deduced and approximated to be

$$
C(\rho, \theta) \cong M \log _{2}\left(\frac{\gamma}{L}\right)+M \log _{2}\left(\frac{R}{d_{m}}\right)^{\alpha}+\frac{1}{\ln 2} \sum_{i=0}^{M-1} \psi(L-i)
$$

From (11) and (13), the expression of downlink ergodic capacity can be deduced as

$$
C(\rho, \theta) \cong K_{1} \log _{2}\left(\frac{\gamma}{L}\right)+K_{1} \log _{2}\left(\frac{R}{d_{m}}\right)^{\alpha}+\frac{1}{\ln 2} \sum_{i=0}^{K_{1}-1} \psi\left(K_{2}-i\right)
$$

Where $K_{1}=\min (M, L)$, and $K_{2}=\max (M, L)$.

Since the above formula is a function of particular MS position $(\rho, \theta)$, the downlink average ergodic capacity considering arbitrary distribution of user is given as 


$$
\bar{C}(\boldsymbol{\mu})=\frac{2 \pi R}{9 P Q} \sum_{p=0}^{P} \sum_{q=0}^{Q} \delta_{p, q} \rho_{p} C\left(\rho_{p}, \theta_{q}\right) f\left(\rho_{p}, \theta_{q}\right)
$$

where $f(\rho, \theta)$ is the probability density function (pdf) of MS; $\boldsymbol{\mu}=\left\{\left(r_{1}, \beta_{1}\right),\left(r_{2}, \beta_{2}\right), \mathrm{L},\left(r_{n}, \beta_{n}\right)\right\}$ represents the set of distributed antenna port coordinate.

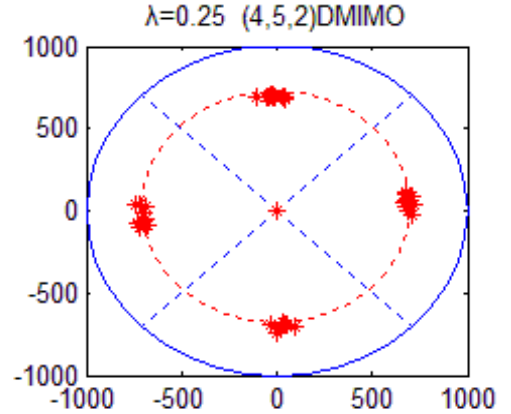

(a) Radius of Cell R(m)

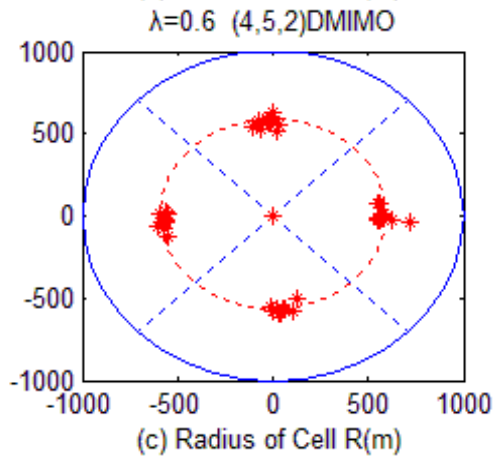

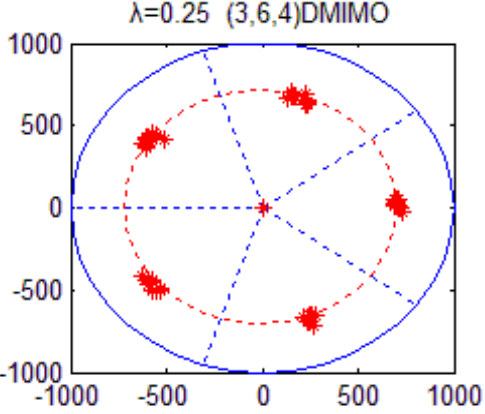

(b) Radius of Cell R(m)

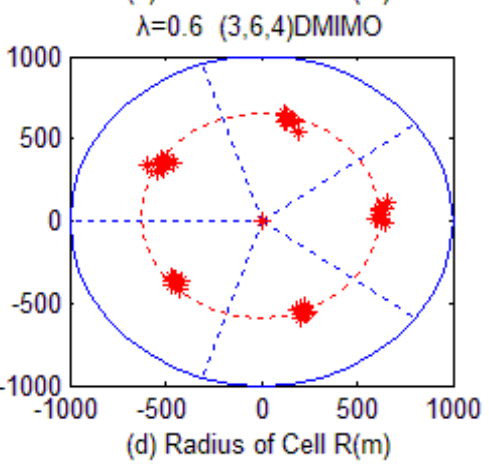

Fig.2. PSO searching results of DMIMO systems with different antenna configuration

\section{Antenna Position Optimization}

We treat the above downlink average ergodic capacity as objective function to improve the DMIMO systems' performance and then to achieve the optimum antenna location coverage. The optimization problem can be equivalent to

$$
\begin{gathered}
\max \bar{C}(\boldsymbol{\mu}) \\
\text { s.t } \boldsymbol{\mu}=\left\{\left(r_{1}, \beta_{1}\right),\left(r_{2}, \beta_{2}\right), \mathrm{L},\left(r_{n}, \beta_{n}\right)\right\}, 0 \leq r_{n} \leq R, 0 \leq \beta_{n} \leq 2 \pi
\end{gathered}
$$

To solve this NP-hard problem, we adopt the so-called particle swarm optimization (PSO) algorithm as the intelligent method to search its global solution due to its strong applicability[11].

This paper adopts fan-shaped region division method to speed up the searching process. After region division and particle initialization, we start to search the optimal antenna port positions in cell of $(4,5,2)$ DMIMO and $(3,6,4)$ DMIMO systems, under the condition of MS being in uniform distribution $(\lambda=0.25)$ and non-uniform distribution $(\lambda=0.6)$.

Fig. 2 shows the searching results of the two different configuration of DMIMO systems applying PSO. In the figure, particle swarm in each region finally concentrates in a fixed position of the fan-shaped region .

\section{Simulation Results and Analysis}

Fig.3 gives some simulation curves of downlink ergodic capacities changing with the radius of antenna ports circle when changing antenna port number $N$ and path loss coefficient $\alpha$. 

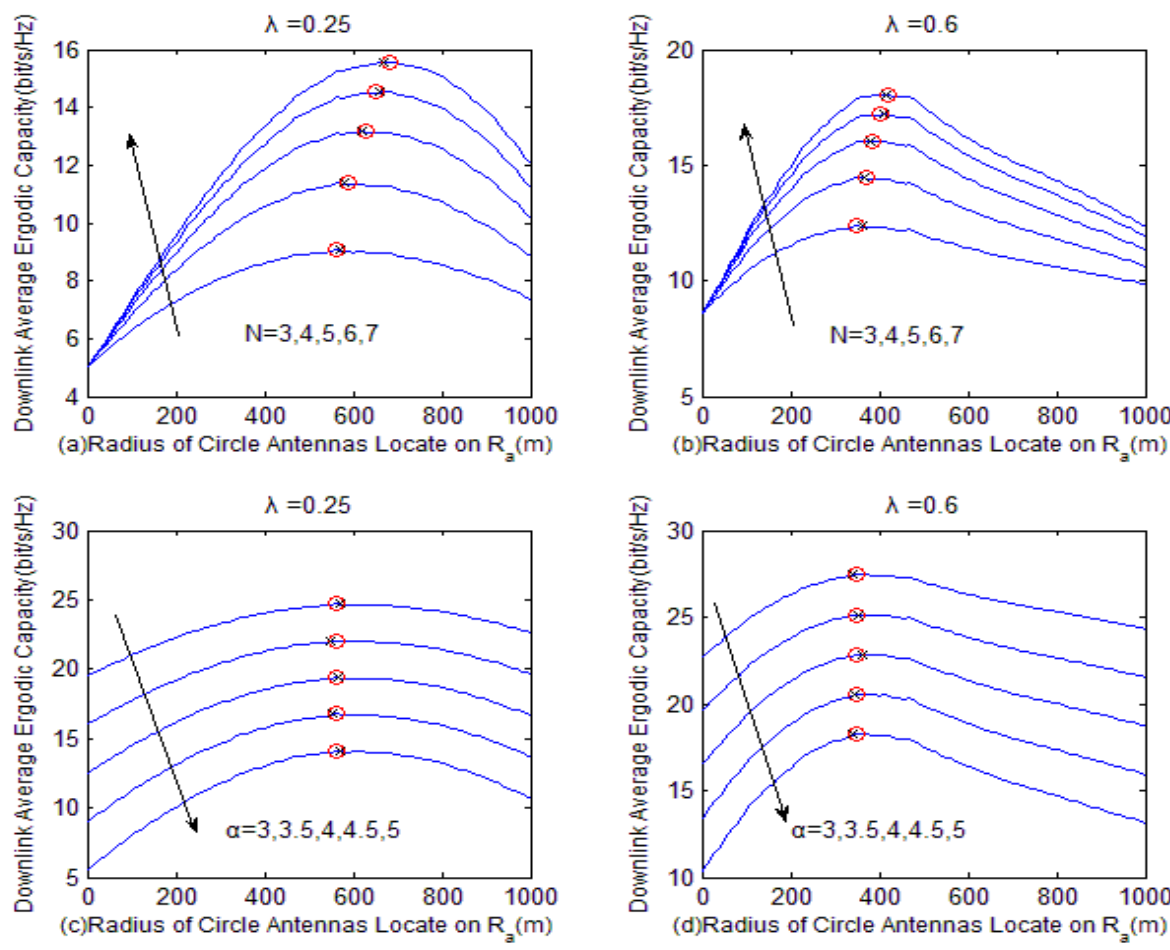

Fig.3. Relationship between mean ergodic capacity and antenna position

In simulation, other parameters are: $\gamma=1 d B, \sigma_{s h}=8 d B, L=3, M=2$. From simulation sub-graphs (a) and (b), we can see that, when antenna port number $N$ is more and more intensive, the average ergodic capacity is also increasing, and the optimal antenna positionis moving to the cell edge. Subgraphs (c) and (d) are simulation curves of (2,4,3) DMIMO systems. We can see that the average ergodic capacity is decreasing according to the change of $\alpha$, but the optimal circle radius is nearly not changed.

In Fig.3, the symbol ' $O$ ' represents the optimal position obtained by computer searching results, and the symbol ' $x$ ' represents the optimal one obtained by PSO searching.It can be shown that the errors between the computerand PSO searching valuesarequite small. So, in order to improve the searching efficiency, PSOalgorithm can be regarded as an idealsuboptimal searching method.

\section{Conclusions}

The optimal antenna port location in circular cell of DMIMO systems based on PSO with maximum downlink average ergodic capacity as an optimization objective is studied. The approximate closed form expression of average ergodic capacity with antenna port selection scheme under higher SNR condition is derived. Then PSO intelligent algorithm is employed to search the optimal antenna port position layout in the case of different DMIMO configurations. It is shown from the computer results that the PSO searching scheme can exactly reflect the actual optimal position of antenna ports. The numerical resultsgiven in this paper can provide a valuable theoretical reference fornew generation mobile communications based on DMIMO technique.

\section{References}

[1] X. Z. Wang,P. C Zhu,M .Chen.Antenna Location Optimization for Circular-Layout Distributed Antenna Systems[J].IEICE transactions on communications, vol.92,no.9, pp. 2980-2983, 2009.

[2] H M Chen, J B,Wang, M Chen. Outage performance of distributed antenna systems over shadowed Nakagami-fading channels[J].European Transactions on Telecommunications, vol. 20,no. 5, pp. 531-535, 2009.

[3] Y. Shen, Y. Tang, T. Kong, and S. Shao. Optimal antennalocation for STBC-OFDM downlink 
with distributed transmit antennas in linear cells,” IEEE Commun. Lett., vol. 11,no. 5, pp.387-389, May 2007.

[4] X.Z.Wang, P.C Zhu, M. Chen. Antenna location design for generalized distributedantenna systems. IEEE Communications Letters, vol. 13,no. 5, pp. 315-317, 2009.

[5] E Park,S R Lee,I Lee. Antenna placement optimization for distributed antenna systems[J]. IEEE Transactions on Wireless Communications, vol. 11,no. 7, pp. 2468-2477, 2012.

[6] J.Y.Wang, J.B Wang and M. Chen. System Capacity Analysis and Antenna PlacementOptimization for Downlink Transmission in DistributedAntenna Systems. Wireless Pers Communications, 2012.

[7] Y Z Qian, M Chen, X Z Wang. Antenna Location Design for Distributed AntennaSystems with Selective Transmission. Wireless Communications and Signal Processing(WCSP), pp.13-15, Nov.2009.

[8] T. K.Y.Lo. Maximum ratio transmission. IEEE Trans. Commun, Vol.47,pp. 1458-1461, Oct. 1999.

[9] D M Wang,X You,J Wang. Spectral efficiency of distributed MIMO cellular systems in a composite fading channel[C]. IEEE International Conference on Communications.(ICC), 1259-1264, 2008.

[10]M Antonia,Tulino and Sergio Verdu. Random Matrix Theory and Wireless Communications, Now Publishers Inc., 2004.

[11]R. Eberhart and J. Kennedy, “A New Optimizer Using Particle Swarm Theory.” 6th International Symposium on Micro-machine and Human Science, pp. 39-43, Oct. 1995. 